\title{
Aberrant Blood Vessel Formation Connecting the Glomerular Capillary Tuft and the Interstitium Is a Characteristic Feature of Focal Segmental Glomerulosclerosis-like IgA Nephropathy
}

\author{
Beom Jin $\operatorname{Lim}^{1,2} \cdot$ Min Ju Kim ${ }^{3}$ \\ Soon Won Hong ${ }^{1} \cdot$ Hyeon Joo Jeong ${ }^{1}$ \\ ${ }^{1}$ Department of Pathology, ${ }^{2}$ Severance Institute \\ for Vascular and Metabolic Research, Yonsei \\ University College of Medicine, Seoul; \\ ${ }^{3}$ Department of Pathology, Gachon University Gil \\ Medical Center, Incheon, Korea \\ Received: January 14, 2016 \\ Revised: January 28, 2016 \\ Accepted: February 1, 2016 \\ Corresponding Author \\ Hyeon Joo Jeong, MD \\ Department of Pathology, Yonsei University College \\ of Medicine, 50-1 Yonsei-ro, Seodaemun-gu, Seoul \\ 03722, Korea \\ Tel: +82-2-2228-1766 \\ Fax: +82-2-362-0860 \\ E-mail: jeong10@yuhs.ac
}

\begin{abstract}
Background: Segmental glomerulosclerosis without significant mesangial or endocapillary proliferation is rarely seen in IgA nephropathy (IgAN), which simulates idiopathic focal segmental glomerulosclerosis (FSGS). We recently recognized aberrant blood vessels running through the adhesion sites of sclerosed tufts and Bowman's capsule in IgAN cases with mild glomerular histologic change. Methods: To characterize aberrant blood vessels in relation to segmental sclerosis, we retrospectively reviewed the clinical and histologic features of 51 cases of FSGS-like IgAN and compared them with 51 age and gender-matched idiopathic FSGS cases. Results: In FSGS-like IgAN, aberrant blood vessel formation was observed in $15.7 \%$ of cases, $1.0 \%$ of the total glomeruli, and $7.3 \%$ of the segmentally sclerosed glomeruli, significantly more frequently than in the idiopathic FSGS cases $(p=.009)$. Aberrant blood vessels occasionally accompanied mild cellular proliferation surrounding penetrating neovessels. Clinically, all FSGS-like IgAN cases had hematuria; however, nephrotic range proteinuria was significantly less frequent than idiopathic FSGS. Conclusions: Aberrant blood vessels in IgAN are related to glomerular capillary injury and may indicate abnormal repair processes in IgAN.
\end{abstract}

Key Words: Glomerulosclerosis, focal segmental; Glomerulonephritis, IgA; Kidney glomerulus; Neovascularization
IgA nephropathy (IgAN) is characterized by dominant or codominant $\operatorname{IgA}$ deposits in the mesangial matrix and is usually accompanied by focal or diffuse mesangial proliferation. However, IgAN has a variety of histologic features ranging from normal to minimal glomerular alterations to diffuse endocapillary and crescentic glomerulonephritis.

Glomerular segmental sclerosis is not unusual in biopsy samples that are diagnosed as IgAN, but it is usually associated with severe mesangial proliferation and/or advanced tubulointerstitial fibrosis. ${ }^{1,2}$ Haas $^{3,4}$ reported a form of segmental sclerosis in IgAN that did not accompany mesangial proliferation and demonstrated clinical features that were indistinguishable from idiopathic focal segmental glomerulosclerosis (FSGS). Of the 18 cases presented in his study, $82 \%$ had nephrotic syndrome and all but one case had favorable prognoses. Another description of segmental sclerosis without significant mesangial proliferation was reported by Weber et al. ${ }^{2}$ In their series of 26 cases, nephrotic syndrome was present in $24 \%$, diffuse effacement of foot pro- cesses in only one case and the clinical outcomes were worse than in cases without FSGS. These two contrasting studies suggest that cases of mild IgAN with FSGS that are morphologically similar but have different pathogeneses may have different clinical outcomes. We recently recognized aberrant blood vessels running through the adhesion sites of sclerosed tufts and Bowman's capsule in IgAN with FSGS features. Since these vessels were present away from the glomerular hilum, they were regarded as related to previous glomerular capillary injury. Glomerular capillary necrosis ${ }^{5}$ may be the forerunner of this lesion, but there have been no detailed descriptions of these vessels in IgAN.

To characterize segmental sclerosis in relation to abnormal vessels in early $\operatorname{Ig} A N$, we retrospectively reviewed clinical and histologic features of FSGS-like IgAN without accompanying severe glomerular cellular proliferation and/or advanced tubulointerstitial fibrosis. Idiopathic FSGS cases were used for comparison. 


\section{MATERIALS AND METHODS}

\section{Materials}

A total of 565 cases with diagnoses of IgAN in native kidneys were retrieved from the renal biopsy registry of the Department of Pathology, Yonsei University Health System, between 1999 and 2008. Among these, 92 cases were diagnosed as IgAN, subclass II, according to the Haas classification. We selected 51 IgAN cases with focal and segmental sclerosis. The exclusion criteria included (1) significant glomerular mesangial proliferation of more than three cells in one mesangial area distant from the vascular hilum in 2-3- $\mu$ m-thick periodic acid-Schiff (PAS)-stained sections; (2) endocapillary proliferation; (3) global glomerulosclerosis or tubular atrophy of $40 \%$ or more; or (4) insufficient sample size of less than six glomeruli on light microscopy. For comparison, age- and gender-matched idiopathic FSGS cases from the same period were collected from our renal biopsy registry. The same exclusion criteria were applied to these cases.

\section{Tissue processing of renal biopsies}

All biopsy samples were examined using light, immunofluorescent, and electron microscopy. For light microscopic examination, 2-3- $\mu \mathrm{m}$-thick sections were stained with hematoxylin and eosin, PAS, aldehyde fuchsin orange $G$, and methenamine silver methods. Two to four tissue sections were mounted per glass slide, and two slides were prepared for each stain, resulting in eight slides for each biopsy. For immunofluorescence examination, snap-frozen 3- $\mu$ m-thick sections were mounted in optimal cold temperature compound and stained with antibodies against IgG, IgA, IgM, C3, C4, C1q, and fibrinogen (Dako Cytomation, Glostrup, Denmark). For electron microscopy, fresh renal tissue was cut at the time of biopsy, and one to three blocks of $1 \mathrm{~mm}^{3}$ renal tissue were double fixed with glutaraldehyde and osmium tetroxide, routinely processed, and stained with uranyl acetate and lead citrate.

\section{Assessment of renal histology}

An average of 26.7 consecutive sections (range, 18 to 32) were examined in each case. All glomeruli in each section were traced. The total glomeruli and glomeruli showing segmental sclerosis were counted. The segmental sclerosis was subtyped in each case as classic, perihilar, tip, cellular or collapsing variants and were numbered. ${ }^{6}$ In segmental sclerosis, capillary adhesion to Bowman's capsule with or without aberrant vessel formation was also counted. To validate aberrant vessels, we followed afferent and efferent arterioles in the vascular poles and confirmed that there were no direct connections between vascular poles and presumed neovessels.

The other histologic parameters that we evaluated were type of glomerular crescent, percentage of global glomerulosclerosis, degree of tubular atrophy, interstitial fibrosis, and interstitial inflammation, and presence of arteriolar hyalinosis and arteriosclerosis. Crescents were defined as extracapillary proliferations of at least two layers that occupied at least $10 \%$ of the circumference. The degrees of tubular atrophy, interstitial fibrosis, interstitial inflammation, and arteriolar hyalinosis were semi-quantitatively scored according to the Banff 97 criteria.

\section{Clinical data}

Patient age, gender, serum creatinine level, hematuria, and proteinuria level at the time of biopsy were obtained from the patient medical records and biopsy requisition forms. Proteinuria was evaluated in 49 FSGS-like IgAN patients and in 45 idiopathic FSGS patients by 24-hour urine collection or random urine protein to creatinine ratios.

\section{Statistical analysis}

Results are expressed as mean \pm standard deviation. Comparisons of the frequencies of segmental sclerosis, tuft adhesion to Bowman's capsule, glomerular hyalinosis, and aberrant vessel formation between FSGS-like IgAN and idiopathic FSGS cases were performed using the Mann-Whitney U test. Tubulointerstitial and vascular changes were compared using Pearson's chi-square test combined with Fisher exact test. p-values less than .05 were considered significant.

\section{RESULTS}

\section{Morphologic features of segmental sclerosis in FSGS-like IgAN}

We observed an average of 16.3 glomeruli in each case of FSGSlike IgAN. Of the 829 total glomeruli, 110 (13.3\%) showed segmental sclerosis. The most common subtype of segmental sclerosis was classic type, followed by tip and perihilar types. Hyalinosis was present in one glomerulus. Glomerular capillary tuft adhesion to Bowman's capsule was observed in 95 glomeruli (86.4\% of the segmentally sclerosed glomeruli) in 49 cases. Among them, nine cases $(8.2 \%$ of the total segmentally sclerosed glomeruli) demonstrated capillary-sized blood vessels connecting the segmentally sclerosed tuft and periglomerular interstitium. These vessels began in the sclerosed tufts, ran perpendicularly through the gap of Bowman's capsule, and merged 
into the capillary networks of the interstitium. The vessels were surrounded by normal-looking glomerular capillary loops in five glomeruli, but accompanied endocapillary proliferations in four glomeruli (Fig. 1).

\section{Comparison of segmental sclerosis between FSGS-like IgAN and idiopathic FSGS}

In age and gender-matched idiopathic FSGS cases, segmental sclerosis was observed in 119 out of 746 glomeruli (16.0\%). When segmental sclerosis of IgAN was compared with that of idiopathic FSGS, the mean percentages of glomeruli with tuft adhesion, aberrant vessel formation, and glomerular crescents were signifi- cantly higher in the FSGS-like IgAN group. Aberrant blood vessels found in FSGS were similar to those seen in IgAN in morphology but were identified in only one glomerulus $(0.8 \%$ of the segmentally sclerosed glomeruli). The mean percentages of glomerular hyalinosis, the degrees of interstitial fibrosis, tubular atrophy, interstitial inflammation, and arteriolar hyalinosis were not significantly different between the two groups (Table 1).

Clinically, hematuria was present in $100 \%$ of the FSGS-like IgAN patients, whereas $51.0 \%$ of the idiopathic FSGS patients had hematuria. Proteinuria was more prevalent and severe in idiopathic FSGS: $91.1 \%$ of patients had proteinuria and $48.9 \%$ were in the nephrotic range, whereas proteinuria was present in
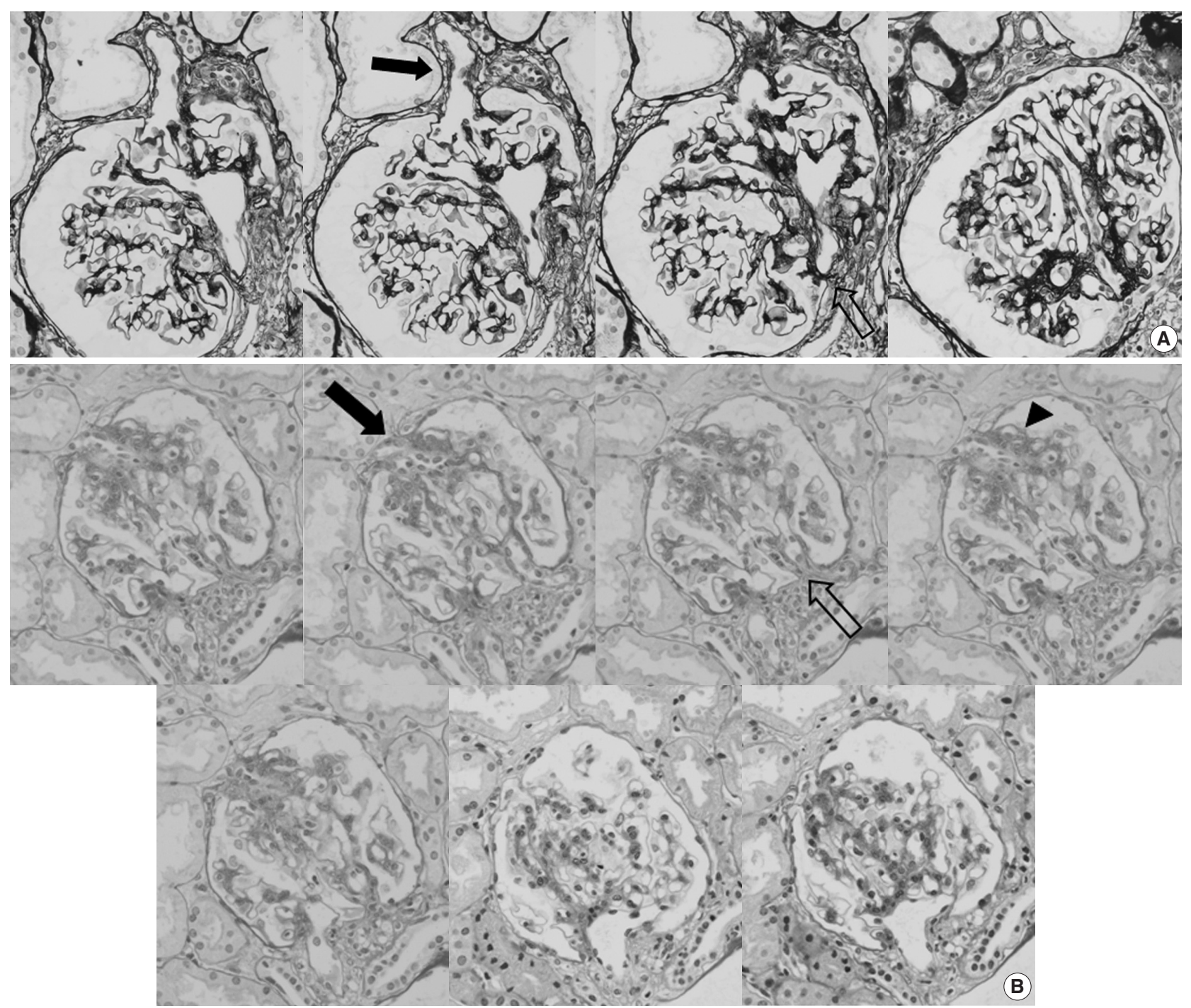

Fig. 1. Consecutive photos of two glomeruli showing extra-efferent vessel formation in focal segmental glomerulosclerosis-like lgA nephropathy. (A, B) Extra vessels connecting the segmentally sclerosed portions of glomerular tufts and the extraglomerular space through the gap of Bowman's capsule were observed when tracing the serial sections. These vessels were not connected to afferent or efferent arterioles in the vascular pole (solid arrows, extra vessels; open arrows, vascular poles). (B) Surrounding glomerular tufts showed capillary and endothelial proliferations in some cases (arrowhead) (periodic acid-Schiff, aldehyde fuchsin orange $\mathrm{G}$, and methenamine silver stain). 
Table 1. Histomorphologic characteristics of FSGS-like IgAN and idiopathic FSGS

\begin{tabular}{|c|c|c|c|}
\hline Variable & FSGS-like lgAN & Idiopathic FSGS & p-value \\
\hline No. of total glomeruli (per case) & $829(16.3)$ & $746(14.6)$ & - \\
\hline Glomeruli with segmental sclerosis, $n(\%)$ & $110(13.3)$ & $119(16.0)$ & - \\
\hline Classic/Perihilar/Tip/Cellular/Collapsing type & $56 / 14 / 42 / 0 / 0$ & 73/7/33/0/6 & - \\
\hline Segmental sclerosis in each case (\%) & $14.6 \pm 7.3$ & $19.5 \pm 16.4$ & $.451^{\mathrm{a}}$ \\
\hline Glomerular hyalinosis in each case (\%) & $0.0 \pm 0.1$ & $0.8 \pm 3.3$ & $.177^{\mathrm{a}}$ \\
\hline Capillary loop adhesion in each case (\%) & $13.2 \pm 7.8$ & $6.5 \pm 8.7$ & $<.001^{\mathrm{a}}$ \\
\hline Neovessel formation in each case (\%) & $1.2 \pm 3.1$ & $0.2 \pm 1.1$ & $.009^{\mathrm{a}}$ \\
\hline Glomerular crescents & & & $<.001^{\mathrm{b}}$ \\
\hline Absent & 39 & 51 & \\
\hline Present & 12 & 0 & \\
\hline Interstitial fibrosis & & & $>.999^{b}$ \\
\hline Absent or mild & 49 & 49 & \\
\hline Moderate to severe & 2 & 2 & \\
\hline Tubular atrophy & & & $.678^{b}$ \\
\hline Absent or mild & 49 & 47 & \\
\hline Moderate to severe & 2 & 4 & \\
\hline \multicolumn{4}{|l|}{ Interstitial inflammation } \\
\hline Absent or mild & 47 & 47 & $>.999^{b}$ \\
\hline Moderate to severe & 4 & 4 & \\
\hline Arteriolar hyalinosis & & & $.463^{b}$ \\
\hline Absent & 39 & 42 & \\
\hline Present & 12 & 9 & \\
\hline
\end{tabular}

FSGS, focal segmental glomerulosclerosis; IgAN, IgA nephropathy.

aMann-Whitney U test; 'Pearson's chi-square test with Fisher exact test.

Table 2. Clinical characteristics of FSGS-like IgAN and idiopathic FSGS

\begin{tabular}{|c|c|c|c|}
\hline Variable & $\begin{array}{c}\text { FSGS-like } \\
\text { IgAN }\end{array}$ & $\begin{array}{l}\text { Idiopathic } \\
\text { FSGS }\end{array}$ & p-value \\
\hline No. of cases & 51 & 51 & \\
\hline Male:Female & $25: 26$ & $24: 27$ & \\
\hline Age, mean (range, yr) & $35.1(7-65)$ & $35.6(7-67)$ & \\
\hline Proteinuria $^{a}$ & 39 (79.9) & $41(91.1)$ & $0.124^{b}$ \\
\hline Nephrotic range & $1(2.0)$ & $22(48.9)$ & $0.000^{b}$ \\
\hline Hematuria $^{\mathrm{a}}$ & $51(100)$ & $26(51.0)$ & $0.000^{b}$ \\
\hline Serum creatinine level (mg/dL) & $1.0 \pm 0.2$ & $1.2 \pm 0.7$ & $0.099^{\circ}$ \\
\hline
\end{tabular}

Values are presented as number (\%) or mean \pm standard deviation unless otherwise indicated.

FSGS, focal segmental glomerulosclerosis; IgAN, IgA nephropathy.

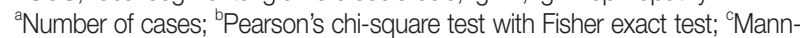
Whitney $\mathrm{U}$ test.

79.9\% of FSGS-like IgAN patients with nephrotic range in $2.0 \%$. Other clinical parameters including mean serum creatinine levels were similar in both groups (Table 2).

\section{DISCUSSION}

Aberrant vessels were more frequently observed in cases of FSGS-like IgAN than in idiopathic FSGS. Otherwise, the morphology of segmental sclerotic glomeruli was indistinguishable in two diseases, and the most common form was the classic type.
Considering that more than 25 sections were examined, these features were rare and may be under-recognized or unnoticed in routine practice. Clinically, our cases were different from those reviewed by Haas ${ }^{3,4}$ but similar to those reviewed by Weber et al. ${ }^{2}$ The frequency of hematuria was higher and that of nephrotic range proteinuria was lower than observed by Haas. ${ }^{3,4}$ Associations of nephrotic range proteinuria have been reported in IgAN with normal histology. However, it has not been resolved whether this entity is a subtype of IgAN or an overlap of IgAN and minimal change disease. Likewise, it is possible that the cases of FSGS-like IgAN described by Haas ${ }^{3,4}$ were subtypes of IgAN or represented the overlap of IgAN and minimal change disease/ FSGS. By contrast, consistent hematuria may suggest the involvement of capillary loop injury in FSGS-like IgAN.

Extra new vessel formation is rarely reported in animal models or in human diabetic nephropathy. Kriz et al. ${ }^{8}$ proposed that "extraterritorial glomerular capillaries" that connect the glomerular space and periglomerular interstitium are responsible for the development of interstitial fibrosis in a hypertensive rat FSGS model. The extra vessels were located in the glomerular hilum in human diabetic nephropathy and did not cause rupture of the capillary loops, and most were connected to the second- and third-order branches of the afferent arteriole and drained into the peritubular capillaries. ${ }^{9}$ The number of extra vessels ranged from 
several to more than 10 per glomerulus. Rare extra vessels have been observed in a variety of cases, also localized within the glomerular hilum. ${ }^{10,11}$ However, the aberrant vessels observed in our study differed from those in diabetic nephropathy in location and numbers. Extra vessels similar to our cases can be seen in glomerulonephritis characterizing small vessel vasculitis ${ }^{5}$ such as Henoch-Schönlein purpura nephritis, antineutrophil cytoplasmic antibody-associated nephritis, and lupus nephritis. In these cases, new vessel formation was observed especially inside or near crescents.

Glomerular capillary loop sclerosis and adhesion were grouped together in a proposed Oxford classification of $\operatorname{IgAN},{ }^{12}$ mainly to increase reproducibility between observers. However, not only are they morphologically distinct but they also differ in pathomechanisms. Glomerular capillary loop sclerosis is characterized by capillary loop collapse and accumulation of scleroprotein inside the glomerular capillary basement membrane. It can be caused by ischemia, toxins, or drugs, but the glomerular basement membrane integrity is essentially preserved. In contrast, glomerular capillary adhesion to Bowman's capsule produces a break in glomerular basement membrane integrity and may develop with or without glomerular capillary rupture. Therefore, adhesions and new vessels in IgAN might be signs of glomerular capillary injury and rupture. The presence of endocapillary proliferation and small glomerular crescents surrounding vessels supports this possibility. Glomerular capillary loop injury may be triggered by immune deposits along the capillary loops; however, our cases had localized mesangial deposits without peripheral capillary IgA deposits. Glomerular capillary basement membranes may be fragile and likely to break without deposits, since glomerular basement membrane thinning or attenuation is frequent in IgAN. ${ }^{13}$ Mesangial cells have been shown to significantly down regulate vascular endothelial growth factor A mRNA and increase inducible nitric oxide synthase mRNA when incubated with aberrantly glycosylated IgA. Therefore, these molecules may be involved in the evolution of abnormal repair in IgAN. ${ }^{14}$ It is unclear how aberrant vessels affect glomerular filtration and nearby structures, but they may contribute to interstitial inflammation and fibrosis by directly delivering pathogenic filtrate to the peritubular capillaries and interstitium. ${ }^{8}$

In conclusion, aberrant blood vessels were observed in segmentally sclerosed glomerular tufts in FSGS-like IgAN. Such structures indicate aberrant repair processes in glomerular capillaries. Further clinical and experimental studies may be needed to elucidate this faulty repair mechanism.

\section{Conflicts of Interest}

No potential conflict of interest relevant to this article was reported.

\section{Acknowledgments}

This study was supported by a grant from the Korean Society of Pathologists to Lim BJ.

\section{REFERENCES}

1. Packham DK, Yan HD, Hewitson TD, et al. The significance of focal and segmental hyalinosis and sclerosis (FSHS) and nephrotic range proteinuria in IgA nephropathy. Clin Nephrol 1996; 46: 225-9.

2. Weber CL, Rose CL, Magil AB. Focal segmental glomerulosclerosis in mild IgA nephropathy: a clinical-pathologic study. Nephrol Dial Transplant 2009; 24: 483-8.

3. Haas M. IgA nephropathy histologically resembling focal-segmental glomerulosclerosis: a clinicopathologic study of 18 cases. Am J Kidney Dis 1996; 28: 365-71.

4. Haas M. Histologic subclassification of IgA nephropathy: a clinicopathologic study of 244 cases. Am J Kidney Dis 1997; 29: 829-42.

5. D'Amico G, Napodano P, Ferrario F, Rastaldi MP, Arrigo G. Idiopathic IgA nephropathy with segmental necrotizing lesions of the capillary wall. Kidney Int 2001; 59: 682-92.

6. Thomas DB, Franceschini N, Hogan SL, et al. Clinical and pathologic characteristics of focal segmental glomerulosclerosis pathologic variants. Kidney Int 2006; 69: 920-6.

7. Solez K, Colvin RB, Racusen LC, et al. Banff 07 classification of renal allograft pathology: updates and future directions. Am J Transplant 2008; 8: 753-60.

8. Kriz W, Hosser H, Hähnel B, Gretz N, Provoost AP. From segmental glomerulosclerosis to total nephron degeneration and interstitial fibrosis: a histopathological study in rat models and human glomerulopathies. Nephrol Dial Transplant 1998; 13: 2781-98.

9. Min W, Yamanaka N. Three-dimensional analysis of increased vasculature around the glomerular vascular pole in diabetic nephropathy. Virchows Arch A Pathol Anat Histopathol 1993; 423: 201-7.

10. Smith JP. Anatomical features of the human renal glomerular efferent vessel. J Anat 1956; 90: 290-2.

11. Murakami T, Kikuta A, Akita S, Sano T. Multiple efferent arterioles of the human kidney glomerulus as observed by scanning electron microscopy of vascular casts. Arch Histol Jpn 1985; 48: 443-7.

12. Working Group of the International IgA Nephropathy Network and the Renal Pathology Society, Roberts IS, Cook HT, et al. The Oxford classification of IgA nephropathy: pathology definitions, 
correlations, and reproducibility. Kidney Int 2009; 76: 546-56.

13. Yoshikawa N, Tanaka R, Iijima K. Pathophysiology and treatment of IgA nephropathy in children. Pediatr Nephrol 2001; 16: 446-57.

14. Amore A, Conti G, Cirina P, et al. Aberrantly glycosylated IgA mol- ecules downregulate the synthesis and secretion of vascular endothelial growth factor in human mesangial cells. Am J Kidney Dis 2000; 36: 1242-52. 